Arthroskopie $2021 \cdot 34: 70-73$

https://doi.org/10.1007/s00142-020-00424-9

Angenommen: 26. November 2020

Online publiziert: 16 . Dezember 2020

๑) Der/die Autor(en) 2020

\author{
Lars Brunnader' $\cdot$ Stefan Greiner ${ }^{2,3} \cdot$ Andreas Voss $^{2,3}$ \\ 'Medizinische Universität Graz, Graz, Österreich \\ ${ }^{2}$ sporthopaedicum, Regensburg/Straubing, Deutschland \\ ${ }^{3}$ Klinik und Poliklinik für Unfallchirurgie, Universitätsklinik Regensburg, Regensburg, Deutschland
}

\title{
Superiore Kapselrekonstruktion mittels langer Bizepssehne bei retrahierter Komplettruptur der Supraspinatussehne und der kranialen Infraspinatussehne
}

culum majus und dem Apex des Humeruskopfes, bei Grad 2 liegt der Stumpf zwischen dem Apex und dem Glenoidrand und bei Grad 3 hinter dem Glenoidrand [4].

\section{Fallbeschreibung}

Der 57-jährige Patient stellte sich mit seit 6 Monaten bestehenden Schmerzen in der linken Schulter vor. Die Schmerzsymptomatik konnte keinem Trauma zugeordnet werden. Eine einmalig e. d. durchgeführte Injektionstherapie (Medikament bei Vorstellung unbekannt) führte zu einer leichten Verbesserung der Beschwerdesymptomatik. Neben Schmerzen, die insbesondere bei Überkopfbewegungen und Belastung bestanden, konnte auch ein Kraftverlust detektiert werden.

Bei der körperlichen Untersuchung ließ sich eine freie aktive sowie passive Beweglichkeit feststellen. Der Nackengriff war vollständig durchführbar, der Schürzengriff konnte bis zum 1. Lendenwirbelkörper ausgeführt werden, Bear-Hug und Speed-Test waren positiv. Die Kraftprüfung nach Janda zeigte eine Kraftminderung des M. supraspinatus (Janda Stufe 3) und des M. infraspinatus (Janda Stufe 4), bei der Testung des M. subscapularis konnte hingegen keine Kraftminderung festgestellt werden.

In der Magnetresonanztomographie (MRT; • Abb. 1) ließ sich eine Komplett- ruptur der Supraspinatussehne und der kranialen Infraspinatussehne objektivieren. Die Rissgröße wurde mit Grad 3 nach Bateman klassifiziert, die Retraktion des Sehnenstumpfes wurde als Grad 3 nach Patte und die trophische Muskelqualität als Grad 3 nach Goutallier eingestuft. Darüber hinaus zeigte sich eine Tendinitis der langen Bizepssehne (LBS). In der Röntgenbefundung der linken Schulter präsentierten sich ein Humeruskopfhochstand und eine Arthrose des Akromioklavikulargelenks.

In Zusammenschau der Befunde war eine Weiterführung der konservativen Therapie aus der Sicht des behandelnden Arztes nicht erfolgversprechend, weshalb man sich gemeinsam mit dem Patienten zur operativen Therapie in Form einer Schulterarthroskopie entschloss, in welcher in erster Linie eine Rekonstruktion der superioren Kapsel mittels LBS-Patch und Readaptation des Infraspinatus, eine Tenotomie der langen Bizepssehne (LBS) und eine subakromiale Dekompression durchgeführt werden sollten.

Der primäre arthroskopische Zugang erfolgte über das posteriore Standardportal, anschließend wurde das anterosuperiore Arbeitsportal gesetzt. Bei der palpatorischen und dynamischen Untersuchung zeigte sich das Pulley-System der Bizepssehne rupturiert. Der radiologische Befund der Supra- und Infraspinatussehne bestätigte sich auch arthroskopisch, des Weiteren war eine deutli- 


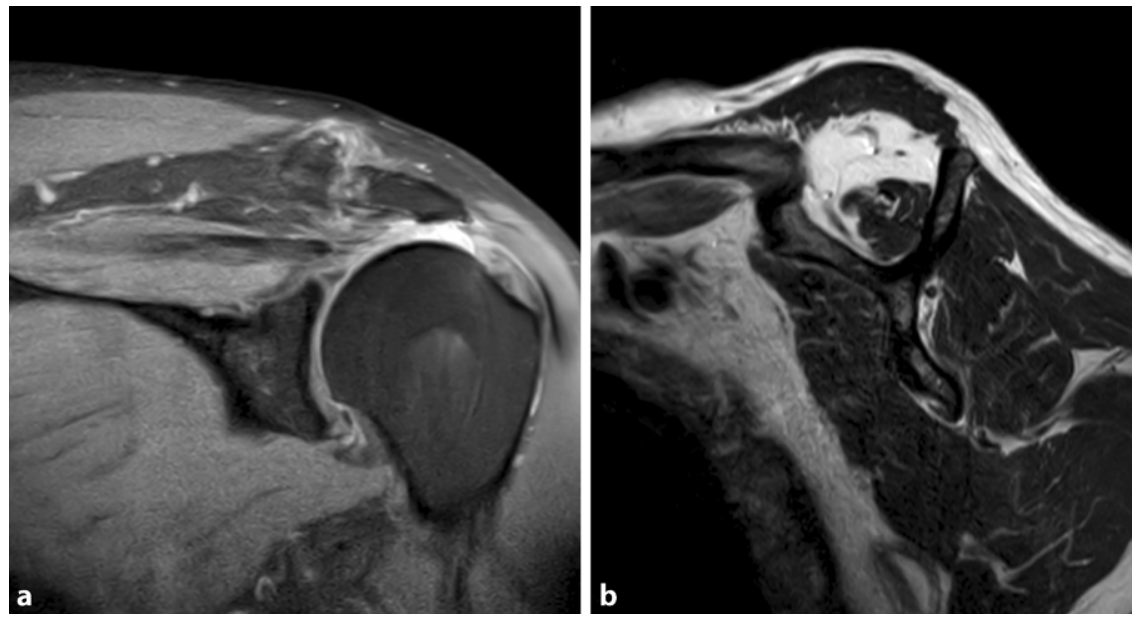

Abb. 1 ॥ Magnetresonanztomographie (MRT) derSchulter. In derT2-gewichteten koronaren Aufnahme (a) ist die Komplettruptur der Supraspinatussehne deutlich zu sehen, während sich in der axialen T1-gewichteten Aufnahme (b) die Ruptur der kranialen Infraspinatussehne objektivieren lässt
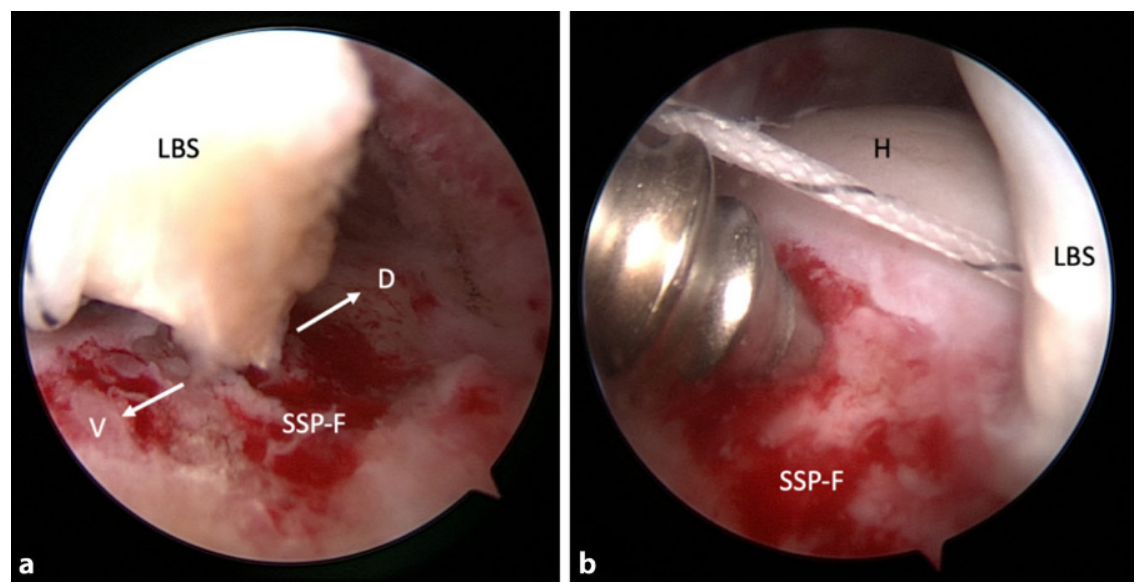

Abb. 2 ム Abgesetzte lange Bizepssehne (LBS) mit ventralem (V) und dorsalem (D) Rand (a), Einbringen eines Fadenankers im Supraspinatus(SSP)-Footprint (b)
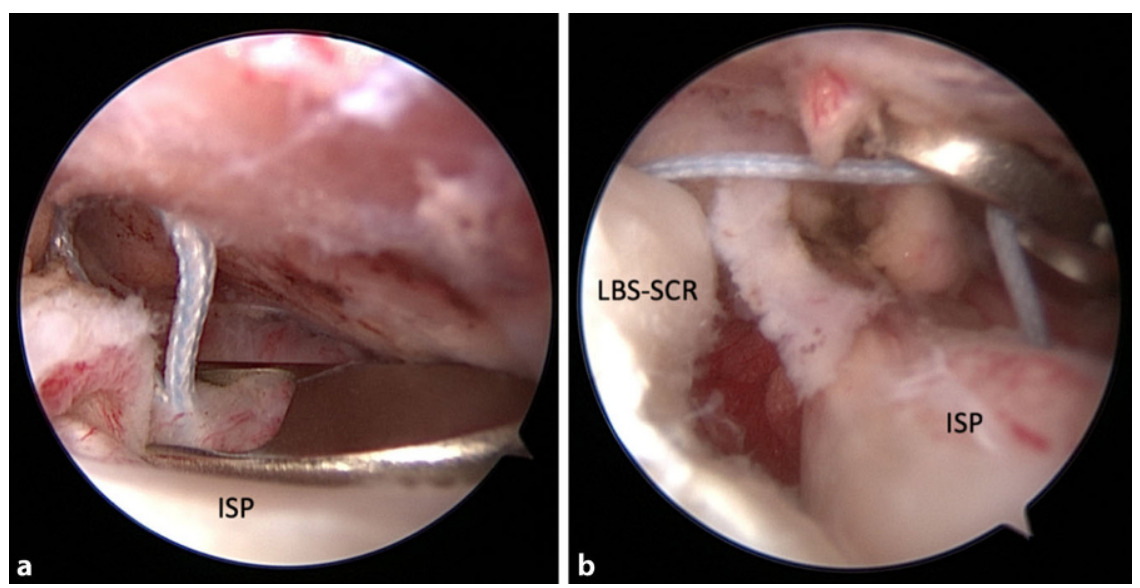

Abb. 3 A Durstechen (a) von langer Bizepssehne (LBS) und Infraspinatussehne (ISP) zur Komplettierung (b) des Verschlusses des posterosuperioren Gelenkraums che Bursitis zu sehen. Anschließend wurde über den posterioren Zugang in den Subakromialraum eingegangen, woraufhin sich auch hier eine deutliche Bursitis zeigte. Anschließend wurden mittels Shaver eine Bursektomie der Bursa subacromialis und eine elektrothermische Blutstillung durchgeführt. Durch die Präzisionsakromioplastik nach Ellmann, welche mithilfe einer rotierenden Fräse umgesetzt wurde, konnte eine gute subakromiale Defilee-Erweiterung erreicht werden. Nach dem erneuten Eingehen in den Gelenkraum erfolgte auch hier zunächst die Bursektomie mit anschließender Blutstillung.

Im nächsten Schritt wurde ein laterales Arbeitsportal gesetzt, wodurch eine Twist-in-Arbeitskanüle (Arthrex $\mathrm{GmbH}$, München, Deutschland) eingebracht wurde. Die folgende Anfrischung des SSP- und ISP-Footprint wurde mit dem Shaver durchgeführt. Hier wurde darauf geachtet, dass dies solange erfolgte bis Blutaustritte aus dem Knochen $\mathrm{zu}$ sehen waren. Auch der superiore Glenoidrand wurde mit dem Shaver präpariert.

Danach wurde die lange Bizepssehne armiert und beim Eintritt in den Sulcus intertubercularis mit zwei FiberWire $^{\circledR}$-Fäden (Arthrex $\mathrm{GmbH}$, München, Deutschland) mittels einer arthroskopischen FastPass Scorpion SL-Zange (Arthrex GmbH, München, Deutschland) durchstochen, woraufhin die lange Bizepssehne distal der Armierung, im Sinne einer Tenotomie, abgesetzt und somit als Autograft für die obere Kapsel vorbereitet wurde. Der nächste Schritt bestand in der Fixierung der langen Bizepssehne mit zwei 5,5-mm-SwiveLock ${ }^{\circledR}$-Ankern (Arthrex $\mathrm{GmbH}$, München, Deutschland), jeweils am ventralen und dorsalen Rand (- Abb. 2).

Mit dem freien FibreWire ${ }^{\circledR}$-Faden wurde die Infraspinatussehne durchstochen und so eine Teilrekonstruktion des ISP durchgeführt. Danach erfolgte die Side-to-side-Naht der langen Bizepssehne durch Verknüpfung mit der kranialen Infraspinatussehne, um so den kompletten Verschluss des posterosuperioren Gelenkraums zu bewerkstelligen (-Abb. 3). 
Siebeneinhalb Monate nach dem Eingriff war jeweils eine schmerzfreie aktive Abduktion und Flexion bis $90^{\circ}$ möglich. Der Schürzengriff konnte bis zum 5. Lendenwirbelkörper ausgeführt werden, während der Nackengriff mit vorgeneigtem Ellenbogen durchgeführt werden konnte.

\section{Diskussion}

Pathologien der Rotatorenmanschette sind für ca. $70 \%$ aller ärztlichen Konsultationen im Rahmen von Schulterschmerzen verantwortlich [5]. Die Inzidenz der Rotatorenmanschettenrupturen steigt mit dem Alter und beträgt bei Personen zwischen dem 60. und 70. Lebensjahr etwa $25 \%$, über dem 80. Lebensjahr hingegen schon über $50 \%$ [6]. Ein Defekt der superioren Kapsel hat biomechanisch eine erhöhte glenohumerale Translation in alle Richtungen zur Folge [7]. Der Verlust dieses stabilisierenden Faktors des Glenohumeralgelenks führt dazu, dass v. a. eine durch den M. deltoideus bedingte Translation nach anterosuperior geschieht, was $\mathrm{zu}$ einer unzureichenden Funktion der Schulter führt.

Ziel der superioren Kapselrekonstruktion (SCR) ist eine Verbesserung der oben beschriebenen Problematik. Durch eine Rezentrierung des Humeruskopfes kann die ursprüngliche Biomechanik wiederhergestellt werden. Mihata et al. leisteten dahingehend Pionierarbeit und bewiesen, dass durch eine SCR die superiore Stabilität der Rotatorenmanschette wieder vollständig hergestellt werden kann [8].

\section{》) Ein Fascia-lata-Allotransplan- tat sollte zumindest $8 \mathrm{~mm}$ Dicke aufweisen}

Die ursprüngliche SCR-Technik wurde von Mihata et al. in Form eines Fascia-lata-Allotransplantats beschrieben, später in Form eines Fascia-lata-Autotransplantats [8]. Aus Überlegungen der kürzeren Operationszeit, der verringerten Invasivität betreffend der Entnahme und der einfacheren Herstellung gewann das Allograft immer mehr an Popularität, obwohl die Gleichwertigkeit der Transplantate noch nicht nachgewiesen ist [9]. Sowohl Fascia-lata-Allotransplantate als auch humane dermale Allotransplantate scheinen in der Lage zu sein, die $\mathrm{Hu}$ meruskopftranslation zu verringern [10]. Die Dicke des gewählten Transplantats ist ein entscheidender Faktor, inwiefern die Biomechanik des Glenohumeralgelenks wiederhergestellt werden kann. So sollte ein Fascia-lata-Allotransplantat zumindest $8 \mathrm{~mm}$ Dicke aufweisen, sodass es die Translation zum Großteil beheben kann [11].

Die im Fallbericht beschriebene Technik der Verwendung der LBS zur Rekonstruktion der superioren Kapsel hat gegenüber der Verwendung eines Fascialata-Autotransplantats den Vorteil, sich einen weiteren Einschnitt zu ersparen und so möglichen Komplikationen, wie z. B. Narben und Hämatombildung, von vorneherein vorzubeugen. Auch diese Technik ist in der Lage, den Humeruskopf zu rezentrieren. Darüber hinaus werden der subakromiale Spitzendruck und die Kontaktfläche von Humerus und Glenoid signifikant verringert [12, 13]. Ebenso konnte bewiesen werden, dass auch das Fortschreiten von Arthrose oder die Atrophie der Muskulatur der Rotatorenmanschette durch dieses Verfahren verlangsamt werden kann [14]. In mehreren Studien konnte zudem eine signifikante Verbesserung des funktionellen Outcomes sowie eine signifikante Abnahme der Schmerzen nachgewiesen werden [15]. Was die lange Bizepssehne als Transplantat für superiore Kapseldefekte zudem noch interessanter macht, ist der Umstand, dass sie eine geeignete Zellquelle für die Regeneration von Sehnengewebe darstellt [16].

Da es bei einigen Patienten neben dem Ruptur der Rotatorenmanschette jedoch auch $\mathrm{zu}$ einer Degeneration und Rissbildung der langen Bizepssehne kommt [15], ist dieses Verfahren nicht immer geeignet.

\section{Fazit für die Praxis}

In dem hier beschriebenen Fall
konnte die Rotatorenmanschetten-
ruptur eines 57-jährigen Patienten

Arthroskopie 2021 $\cdot 34: 70-73$

https://doi.org/10.1007/s00142-020-00424-9

○ Der/die Autor(en) 2020

L. Brunnader · S. Greiner · A. Voss

\section{Superiore}

Kapselrekonstruktion mittels

langer Bizepssehne bei

retrahierter Komplettruptur

der Supraspinatussehne und

der kranialen

Infraspinatussehne

\section{Zusammenfassung}

In diesem Beitrag berichten die Autoren über einen Patienten mit einer Ruptur der Rotatorenmanschette, welche sich als retrahierte Komplettruptur der Supraspinatussehne und der kranialen Infraspinatussehne präsentierte. Nach

7-monatiger Schmerzpersistenz und anhaltendem Funktionsverlust wurde im Rahmen eines arthroskopischen Eingriffs eine Rekonstruktion der superioren Kapsel durchgeführt. Die lange Bizepssehne wurde hierzu als Autograft verwendet und mit der kranialen Infraspinatussehne verknüpft. Auf diese Weise konnte ein kompletter Verschluss des posterosuperioren Gelenkraums erreicht werden.

Schlüsselwörter

Rotatorenmanschettenruptur · Supraspinatussehne - Infraspinatussehne - Transplantat . Magnetresonanztomographie

\section{Superior capsule reconstruction using the long biceps tendon for retracted complete rupture of the supraspinatus tendon and the cranial infraspinatus tendon}

\section{Abstract}

This article reports the case of a patient with a massive rupture of the rotator cuff, which presented as a retracted complete rupture of the supraspinatus tendon and the cranial infraspinatus tendon. After 7 months of persisting pain and continued loss of function, the superior capsule was reconstructed within the framework of an arthroscopic intervention. The long biceps tendon was used as an autograft and linked to the cranial infraspinatus tendon. In this way it was possible to achieve a complete closure of the posterosuperior joint space.

Keywords

Rotator cuff tear - Supraspinatus tendon . Infraspinatus tendon · Autograft · Magnetic resonance imaging 
erfolgreich mittels superiorer Kapselrekonstruktion behandelt werden.

- Hierzu wurde die lange Bizepssehne als Autograft verwendet und mit der kranialen Infraspinatussehne verknüpft.

- Mittels dieser Technik konnten Biomechanik und Stabilität des Schultergelenks konnten wiederhergestellt werden.

- Die lange Bizepssehne eignet sich, sofern sie keine Degeneration oder Rissbildung aufweist, aufgrund ihrer Eigenschaften prinzipiell sehr gut als Transplantat.

\section{Korrespondenzadresse}

\section{Lars Brunnader}

Medizinische Universität Graz

Graz, Österreich

lars.brunnader@gmail.com

Funding. Open access funding provided by Medical University of Graz.

\section{Einhaltung ethischer Richtlinien}

Interessenkonflikt. L. Brunnader, S. Greiner und A. Voss geben an, dass kein Interessenkonflikt besteht.

Für diesen Beitrag wurden von den Autoren keine Studien an Menschen oder Tieren durchgeführt. Für die aufgeführten Studien gelten die jeweils dort angegebenen ethischen Richtlinien. Für Bildmaterial oder anderweitige Angaben innerhalb des Manuskripts, über die Patienten zu identifizieren sind, liegt von innen und/oder ihren gesetzlichen Vertretern eine schriftliche Einwilligung vor.

Open Access. Dieser Artikel wird unter der Creative Commons Namensnennung 4.0 International Lizenz veröffentlicht, welche die Nutzung, Vervielfältigung, Bearbeitung, Verbreitung und Wiedergabe in jeglichem Medium und Format erlaubt, sofern Sie den/die ursprünglichen Autor(en) und die Quelle ordnungsgemäß nennen, einen Link zur Creative Commons Lizenz beifügen und angeben, ob Änderungen vorgenommen wurden.

Die in diesem Artikel enthaltenen Bilder und sonstiges Drittmaterial unterliegen ebenfalls der genannten Creative Commons Lizenz, sofern sich aus der Abbildungslegende nichts anderes ergibt. Sofern das betreffende Material nicht unter der genannten Creative Commons Lizenz steht und die betreffende Handlung nicht nach gesetzlichen Vorschriften erlaubt ist, ist für die oben aufgeführten Weiterverwendungen des Materials die Einwilligung des jeweiligen Rechteinhabers einzuholen.

Weitere Details zur Lizenz entnehmen Sie bitte der Lizenzinformation auf http://creativecommons.org/ licenses/by/4.0/deed.de.

\section{Literatur}

1. Bakhsh W, Nicandri G (2018) Anatomy and Physical Examination of the Shoulder. Sports Med Arthrosc 26(3):e10-e22

2. Pandey V, Jaap Willems W (2015) Rotator cuff tear: A detailed update. Asia Pac J Sport Med Arthrosc Rehabil Technol 2(1):1-14. https://doi. org/10.1016/j.asmart.2014.11.003

3. Plachel F, Moroder P, Gerhardt C, Scheibel M (2017) Anterosuperiore Rotatorenmanschettenläsion beim jungen Patienten: Ruptur der Subskapularis- und Supraspinatussehne. Arthroskopie 30(3):216-222

4. Zeichen J, Bosch U, Krettek C (2003) Rotatorenmanschette. Trauma Berufskrankh 5(0):s120-s125

5. Piper CC, Hughes AJ, Ma Y, Wang H, Neviaser AS (2018) Operative versus nonoperative treatment for the management of full-thickness rotator cuff tears: a systematic review and meta-analysis. J Shoulder Elbow Surg 27(3):572-576. https://doi. org/10.1016/j.jse.2017.09.032

6. Edwards P, Ebert J, Joss B, Bhabra G, Ackland T, Wang A (2016) Exercise rehabilitation in the non-operative management of rotator cuff tears: a review of the literature. Int J Sports Phys Ther 11(2):279-301 (Available from: http://www.ncbi. nlm.nih.gov/pubmed/27104061\%0A)

7. Ishihara Y, Mihata T, Tamboli M, Nguyen L, Park KJ, McGarry MH et al (2014) Role of the superior shoulder capsule in passive stability of the glenohumeral joint. J Shoulder Elbow Surg 23(5):642-648. https://doi.org/10.1016/j.jse. 2013.09.025

8. Mihata T, McGarry MH, Pirolo JM, Kinoshita M, Lee TQ (2012) Superior capsule reconstruction to restore superior stability in irreparable rotator cuff tears: a biomechanical cadaveric study. Am J Sports Med 40(10):2248-2255

9. Makovicka JL, Chung AS, Patel KA, Deckey DG, Hassebrock JD, Tokish JM (2020) Superior capsule reconstruction for irreparable rotator cuff tears: a systematic review of biomechanical and clinical outcomes by graft type. J Shoulder Elbow Surg 29(2):392-401. https://doi.org/10.1016/j.jse. 2019.07.005

10. Mihata T, Bui CNH, Akeda M, Cavagnaro MA, Kuenzler M, Peterson AB et al (2017) A biomechanical cadaveric study comparing superior capsule reconstruction using fascia lata allograft with human dermal allograft for irreparable rotator cuff tear. J Shoulder Elbow Surg 26(12):2158-2166. https://doi.org/10.1016/j.jse.2017.07.019

11. Mihata T, McGarry MH, Kahn T, Goldberg I, Neo M, Lee TQ (2016) Biomechanical effect of thickness and tension of fascia Lata graft on glenohumeral stability for superior capsule reconstruction in irreparable Supraspinatus tears. Arthroscopy 32(3):418-426. https://doi.org/10.1016/j.arthro. 2015.08.024

12. Han $\mathrm{F}$, Kong $\mathrm{CH}$, Hasan $\mathrm{MY}$, Ramruttun $\mathrm{AK}$, Kumar VP (2019) Superior capsular reconstruction for irreparable supraspinatus tendon tears using the long head of biceps: a biomechanical study on cadavers. Orthop Traumatol Surg Res 105(2):257-263. https://doi.org/10.1016/j.otsr. 2018.10.023

13. Han SY, Lee TQ, Wright DJ, Park IJ, Mauro M, McGarry MH et al (2020) Effect of biceps rerouting technique to restore glenohumeral joint stability for large irreparable rotator cuff tears: a cadaveric biomechanical study. J Shoulder Elbow Surg 29(7):1425-1434. https://doi.org/10.1016/j.jse. 2019.11.015
14. Mihata T, Lee TQ, Watanabe C, Fukunishi K, Ohue M, Tsujimura T et al (2013) Clinical results of arthroscopic superior capsule reconstruction for irreparable rotator cuff tears. Arthroscopy 29(3):459-470. https://doi.org/10.1016/j.arthro. 2012.10.022

15. Veen EJD, Stevens M, Diercks RL (2018) Biceps autograft augmentation for rotator cuff repair: a systematic review. Arthroscopy 34(4):1297-1305. https://doi.org/10.1016/j.arthro.2017.10.044

16. Pietschmann MF, Wagenhäuser MU, Gülecyüz MF, Ficklscherer A, Jansson V, Müller PE (2014) The long head of the biceps tendon is a suitable cell source for tendon tissue regeneration. Arch Med Sci 10(3):587-596 